\title{
Mitral valve repair: a clinical and echocardiographic study
}

\author{
Minsi Xu, David J McHaffie, Allan D Hilless
}

Objective-To evaluate the clinical and echocardiographic results of mitral valve repair done within an 11 year period.

Design-Retrospective review of case notes and clinical and echocardiographic examination of survivors. Analysis was made according to the intention to treat principle.

Patients-A consecutive series of 94 patients with mitral valve disease who had mitral reconstruction between 1980 and 1991.

Interventions-Mitral repair according to the techniques of Carpentier or Duran.

Main outcome measures-Operative mortality, actuarial survival, rates of freedom from reoperation, thromboembolism, infective endocarditis, clinical state, and echocardiographic findings. Results-There were equal numbers of males and females (mean (range) age 49 (4-74) years). The aetiology of mitral disease was degenerative in $59 \%$ and rheumatic in $30 \%$ of the patients. Operative mortality was $3 \%$. The 10 year actuarial and valve related survival rates were $67 \%$ and $75 \%$. At 10 years, $84 \%$ of the patients were free from thromboembolism, $94 \%$ free from haemorrhage related to anticoagulation, $88 \%$ free from infective endocarditis, and $73 \%$ free from reoperation. Of 75 survivors with completed follow up, 96\% were in New York Heart Association functional class I or II and $64 \%$ were in sinus rhythm. Of 55 survivors who had an echocardiogram at follow up, normal left ventricular end diastolic dimension was shown in $76 \%$ and normal left ventricular shortening fraction in $89 \%$. Doppler studies showed no mitral regurgitation in $36 \%$, trivial or mild in $42 \%$, moderate in $14 \%$, and severe in $7 \%$ of patients. In stepwise logistic analysis previous myocardial infarction was associated with poor outcome (odds ratio: $13 \cdot 7, \mathrm{p}<0 \cdot 05$ ).

Conclusion-The results are comparable with similar studies and support the value of mitral valve repair. There is reservation about the use of repair for patients with previous myocardial infarction.

(Br Heart f 1994;71:51-56)

Mitral valve repair has been reported to give excellent results in patients with severe valve disease, especially those with mitral regurgitation. ${ }^{1-3}$ Successful surgery corrects regurgitation or relieves obstruction and is associated with low mortality. Compared with valve replacment, mitral valve repair has reduced risk of thromboembolism and haemorrhage related to anticoagulants. The selection of patients who are most likely to benefit from mitral repair and the long-term durability of the reconstituted valve are important aspects of this treatment. The purpose of our study was to evaluate the long-term results of mitral valve repair and to identify the risk factors associated with poor outcome from the operation.

\section{Patients and methods}

PATIENTS

The study population was a consecutive series of all patients who had mitral valve repair between April 1980 and May 1991. During this period mitral repair was done by one surgeon.

SURGICAL TECHNIQUES AND ANALYSIS OF THE VALVE

Most patients were operated on with moderate systemic hypothermia to $28^{\circ} \mathrm{C}$ until cold crystalloid cardioplegia was achieved. More recently normothermia and continuous warm retrograde blood cardioplegia were used for myocardial preservation. After visual inspection of the mitral valve and determination of the mechanism of incompetence, standard Carpentier techniques of valve reconstruction were used. ${ }^{14}$ Mostly Carpentier rings and some Duran annuloplasty rings were used for annular remodelling, leaflet resection and chordal shortening for leaflet prolapse, and commissurotomy for restricted leaflet motion. At the end of the reconstruction the adequacy of repair was assessed by gently instilling saline into the left ventricular cavity and ensuring that there was apposition of at least $3 \mathrm{~mm}$ of leaflet tissue anteriorly and posteriorly. Checks were made that the line of closure of the anterior and posterior leaflets was parallel to the annuloplasty ring and that there was no puckering in the line of leaflet coaption. Valve function was reviewed during weaning from bypass. The direct left atrial pressure pulse was examined for a regurgitant wave or significant diastolic pressure gradient that would have indicated that too small an annuloplasty ring had been used.

The aetiology of the mitral valve disease was determined by the clinical history and by 
the pathological findings at operation. The nature of valve lesions was classified by the functional and anatomical approaches recommended by Deloche et al (the Carpentier technique) and Cooper et al. ${ }^{12}$

\section{ANTICOAGULATION}

All patients were given warfarin for six weeks to three months after the repair. This medication was continued on a long-term basis for most patients who had atrial fibrillation or a history of preoperative thromboembolism. The dose of warfarin was adjusted by monitoring prothrombin time and, more recently, the international normalised ratio, with the aim of keeping the ratio between two and three times that of the normal control.

\section{FOLLOW UP METHODS}

The preoperative and perioperative information was collected from hospital records. One patient's operation notes could not be found and these data were excluded from the analysis of surgical findings and techniques. Postoperative follow up data were gathered during a 12 month interval (December 1991 to November 1992). Local survivors were reviewed in the outpatient clinic and had an echocardiogram. Patients living in other regions were followed up by experienced clinicians. Where possible an echocardiogram was arranged. Patients unable to be seen by a doctor were contacted by telephone.

\section{ECHOCARDIOGRAPHY}

Cross sectional echocardiograms, including Doppler scans after 1984, were analysed by methods previously described. ${ }^{5}$ Postoperative mitral valve area was calculated from the Doppler mitral valve pressure half time. The degree of mitral regurgitation was determined by Doppler. With trivial or mild regurgitation the regurgitant jet was confined to the proximal third of the left atrium. With moderate regurgitation the jet was detected beyond the proximal third but within the proximal two thirds of the left atrium. With severe regurgitation the jet extended beyond the proximal two thirds of the left atrium.

\section{STATISTICAL ANALYSIS}

The software package used for the statistical analysis was SAS/STAT (SAS Institute Inc. USA). Continuous variables are presented as mean (SD) and percentage is given with $95 \%$ confidence intervals ( $95 \% \mathrm{CIs}$ ). Comparisons between continuous variables were made by Student's $t$ test. Discrete data were compared by $\chi^{2}$ analysis or Fisher's exact test. Actuarial survival and freedom from postoperative complications were calculated with the Kaplan and Meier method. Significance of differences between subgroups was determined by the log rank test. With the intention to treat principle, patients who had reoperation were not excluded from further analysis of valve related events. A backwards stepwise logistic model was used for identification of the preoperative factors associated with poor outcome. Patients with poor outcome included those who died of a valve related cause and survivors with left ventricular enlargement of $\geqslant 65 \mathrm{~mm}$ at least 5 years after the operation. Patients who did not undergo a follow up echocardiogram were excluded from the multivariate analysis.

\section{Results}

PATIENTS

Between April 1980 and May 1991, of 326 patients with mitral regurgitation who had mitral surgery, 223 had primary mitral valve replacement, nine had open mitral valvotomy, and 94 had mitral valve repair. The mean (range) age of the patients who had repairs was 49 (4-74) years. There were equal numbers of males and females. We were able to obtain follow up information on 92 patients $(98 \%)$ of whom 75 were alive at the end of the study. Two patients who had gone overseas were excluded from further analysis. Thirty five survivors who live locally $(47 \%$ of 75$)$ were reviewed and had an echocardiogram. Of the remaining 40 survivors who live in remote regions, 35 were seen by their cardiologists or physicians, three were examined clinically by general practitioners, and two were only contacted by telephone. Echocardiographic information was obtained from 28 of 40 patients who live in other regions. The mean (range) duration of follow up was $68(3-142)$ months.

\section{PREOPERATIVE CHARACTERISTICS}

Table 1 shows the clinical characteristics of the 94 patients. In all patients with pure mitral regurgitation, the degree of regurgitation was moderate or severe. Of the 16 patients with mitral regurgitation and stenosis, $88 \%$ had moderate or severe regurgitation. Two patients with a previous mitral valvotomy had mild regurgitation. Ninety five per cent of the patients were in New York Heart Association (NYHA) functional class III or IV. Five patients had electrocardiographic evidence of previous myocardial infarction.

OPERATIVE FINDINGS AND REPAIR PROCEDURES Table 2 shows the aetiology of the mitral valve disease and table 3 shows the nature of valve lesions. Table 4 shows the techniques of repair and table 5 the associated cardiac procedures. One hundred and twenty nine

Table 1 Preoperative characteristics of 94 patients with mitral valve repair 1980-91

\begin{tabular}{lc}
\hline Characteristic & No(\%) \\
\hline Male & $47(50)$ \\
NYHA functional class: & 0 \\
I & $4(4)$ \\
II & $70(74)$ \\
III & $19(20)$ \\
IV & $47(50)$ \\
Atrial fibrillation & $28(30)$ \\
Coronary heart disease & $4(4)$ \\
History of cerebrovascular events & $2(2)$ \\
Previous mitral valvotomy & $78(83)$ \\
Mitral regurgitation & $16(17)$ \\
Mitral regurgitation and stenosis & $49(4-74)$ \\
Age (mean (range) yr) &
\end{tabular}


Table 2 Aetiology of mitral valve disease in 94 patients with mitral valve repair (1980-91)

\begin{tabular}{lc}
\hline Aetiology & $\mathrm{No}(\%)$ \\
\hline Degenerative & $57(59)$ \\
Rheumatic & $28(30)$ \\
After endocarditis & $5(5)$ \\
Ischaemic & $2(2)$ \\
Congenital & $2(2)$ \\
\hline
\end{tabular}

repairs were done on 93 valves and there were 81 ring annuloplasties. One of 28 rheumatic valves and 44 of 56 myxomatous valves had leaflet resection.

\section{OPERATIVE MORTALITY}

Three patients died within 30 days of the repair, a hospital mortality of $3 \%$ (95\% CI $1 \%$ to $9 \%$ ). All of the operative deaths occurred in patients aged over 60 and happened during the first four years of the study period. Table 6 shows the causes of death. One patient with acute myocardial infarction resulting in papillary muscle dysfunction died at operation from deterioration of left ventricular function. Another patient with mitral regurgitation secondary to gross left ventricular dysfunction caused by severe coronary artery disease died of septicaemia 11 days after the operation. One patient had an embolic stroke shortly after the operation and died 9 days after the repair.

LATE SURVIVAL

There were 14 late deaths in 89 patients with complete follow up. Table 6 shows the causes of death. Twelve deaths were considered to be valve related. The actuarial survival rate, including operative deaths, was $90 \%$ (95\% CI $83 \%$ to $96 \%)$ at 5 years and $67 \%(95 \%$ CI $49 \%$ to $84 \%$ ) at 10 years (fig 1 ). There was no significant difference in survival rate between the group with rheumatic disease and the group with degenerative disease $(73 \% v 64 \%$ at 10 years, $\mathrm{p}>0.05)$. The 15 valve related deaths (including operative deaths) give a survival rate of $90 \%(95 \% \mathrm{CI}$ $83 \%$ to $96 \%)$ at 5 years and $75 \%(95 \%$ CI $62 \%$ to $88 \%$ ) at 10 years.

\section{REOPERATION}

Ten patients (six with rheumatic and four with degenerative disease) needed reoperation. Table 7 shows the causes of reoperation and the surgical findings. Severe recurrent

Table 3 Functional and anatomical classification of valve lesion in $93^{*}$ patients with mitral valve repair 1980-91

\begin{tabular}{|c|c|c|c|}
\hline Functional classification & $\mathrm{No}(\%)$ & Major anatomical lesion & $\mathrm{No}(\%)$ \\
\hline I Normal leaflet motion & $11(12)$ & $\begin{array}{l}\text { Dilated annulus } \\
\text { Perforated leaifet }\end{array}$ & $\begin{array}{l}8(9) \\
3(3)\end{array}$ \\
\hline II Leaflet prolapse: & $76(82)$ & $\begin{array}{l}\text { Ruptured chordae } \\
\text { Elongated chordae }\end{array}$ & $\begin{array}{l}45(48) \\
31(33)\end{array}$ \\
\hline $\begin{array}{l}\text { Anterior leaflet prolapse } \\
\text { Posterior leaflet prolapse } \\
\text { Both leaflet prolapse }\end{array}$ & $\begin{array}{l}22(23) \\
33(35) \\
21(23)\end{array}$ & & \\
\hline III Restricted leaflet motion & $6(6)$ & Commissural fusion & $6(6)$ \\
\hline $93(100)$ & $93(100)$ & & \\
\hline
\end{tabular}

Table 4 Repair procedures done in $93^{*}$ patients with mitral valve repair 1980-91

\begin{tabular}{lc}
\hline Procedures & No(\%) \\
\hline Ring annuloplasty & $81(87)$ \\
Leaflet resection & $49(52)$ \\
Chordal shortening & $46(48)$ \\
Chordal sliding & $25(27)$ \\
Chordal transposition & $4(4)$ \\
Leaflet mobilisation & $6(6)$ \\
\hline ॠOne patient's operation record was missing. \\
†Average number of techniques/patient was $2 \cdot 3$
\end{tabular}

Table 5 Associated procedures in 94 patients with mitral valve repair 1980-91

\begin{tabular}{lc}
\hline Associated procedures & No(\%) \\
\hline Coronary artery bypass graft & $17(18)$ \\
Aortic valve replacement & $11(12)$ \\
Tricuspid annuloplasty & $3(3)$ \\
Atrial septal defect or PFO closure & $2(2)$ \\
Total & $33(35)$ \\
\hline
\end{tabular}

PFO, patent foramen ovale.

Table 6 Causes of death in 94 patients with mitral valve repair 1980-91

\begin{tabular}{llc}
\hline Causes of death & Operative & Late \\
\hline Related to valve $(\mathrm{n}=15):$ & & \\
$\quad$ Sudden death & 1 & 3 \\
Heart failure & 1 & 1 \\
Cerebrovascular accident & 1 \\
Haemorrhage related to anticoagulant & 1 & 1 \\
Septicaemia & & 2 \\
Haemorrhage after valve replacement & & 3 \\
Unknown & & 1 \\
Not related to valve $(\mathrm{n}=2):$ & & 1 \\
$\quad$ Myocardial infarction & & 14 \\
Disseminated intravascular coagulation & &
\end{tabular}

mitral regurgitation was the major cause for both early (within two years of the repair) and late reoperation. Nine patients needed the mitral valve to be replaced and one needed a second repair. Three patients who had the valve replaced died after reoperation. The ten year actuarial rate of freedom from reoperation was $73 \%$ (95\% CI $52 \%$ to $94 \%$, fig 2$)$. The actuarial rate of freedom from reoperation was $79 \%$ in the degenerative group $v$ $56 \%$ in the rheumatic group at 10 years $(p>$ $0 \cdot 05)$.

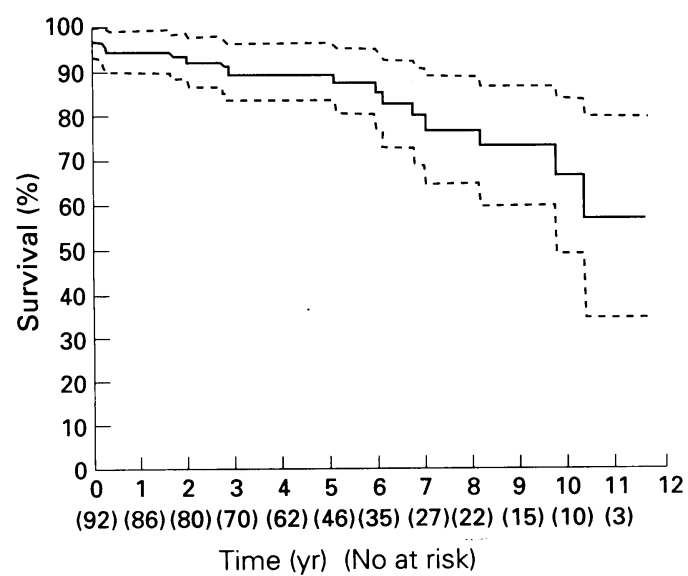

Figure 1 Actuarial survival of 92 patients who had mitral valve repair 1980-91. Broken lines are 95\% CI. 
Table 7 Causes of reoperation and reoperative findings in 94 patients with mitral valve repair 1980-91

\begin{tabular}{lll}
\hline & $N o$ & \\
\cline { 3 - 3 } & $\begin{array}{l}\text { Early reoperation } \\
(n=6,<2 ~ y r)\end{array}$ & $\begin{array}{l}\text { Late reoperation } \\
(n=4,>2 \text { yr })\end{array}$ \\
\hline Causes: & & 2 \\
$\quad$ Residual or recurrent mitral regurgitation & 5 & 1 \\
$\quad$ Infective endocarditis & 1 & 1 \\
$\quad$ Recurrent mitral regurgitation and stenosis & 2 & 2 \\
$\quad$ Reoperative findings: & 2 & 2 \\
$\quad$ Recurrent prolapse or chordae rupture & 2 & \\
$\quad$ Suture detraction or valve restenosis & & \\
\hline
\end{tabular}

THROMBOEMBOLISM

Seven patients had a thromboembolic event. All had atrial fibrillation and two patients were not taking warfarin. Of the four patients with a major episode, three developed sudden loss of consciousness and one had sudden dysphasia and hemiplegia. Three of them made a good recovery; one had residual hemiplegia. The remaining three patients had minor attacks, including episodes of dysphasia or embolism in a peripheral artery. The 10 year actuarial rate of freedom from thromboembolism was $84 \%$ (95\% CI $68 \%$ to $99 \%$, fig 3 ).

\section{HAEMORRHAGE RELATED TO}

ANTICOAGULATION

Forty five per cent of the 92 patients were on long-term anticoagulation. Six patients had complications related to anticoagulation. Three patients, including two who underwent valve replacement, died of gross haemorrhage. Of the other three patients, one had a cerebral haemorrhage with residual dysphasia and two had minor rectal bleeding. The 10 year actuarial rate of freedom from haemorrhage resulting from anticoagulation was $94 \%$ (95\% CI $88 \%$ to $99 \%$ ).

\section{INFECTIVE ENDOCARDITIS}

Valvar endocarditis occurred in three patients (6 months to 10 years after operation). One patient developed endocarditis after the initial mitral repair and the other two had infection after reoperation. All three patients were treated by valve replacement. The actuarial rate of freedom from infective endocarditis was $88 \%$ (95\% CI $69 \%$ to $100 \%$ ) at 10 years.

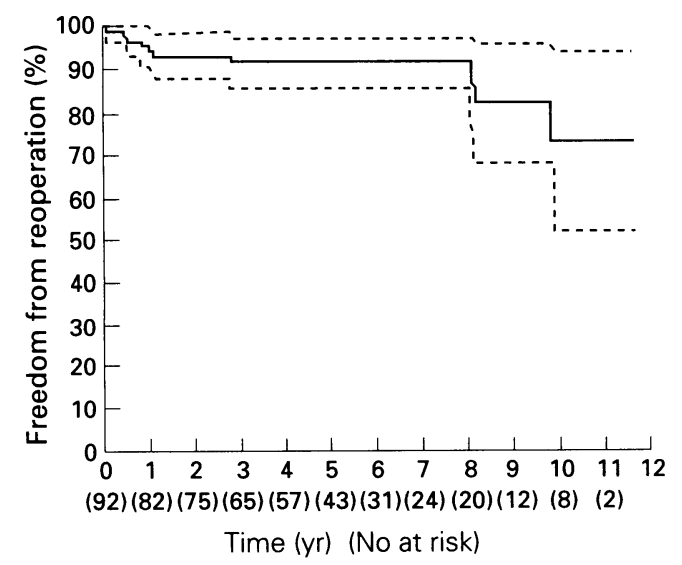

Figure 2 Actuarial freedom from reoperation of 92 patients who had mitral valve repair 1980-91. Broken lines are $95 \%$ CI.

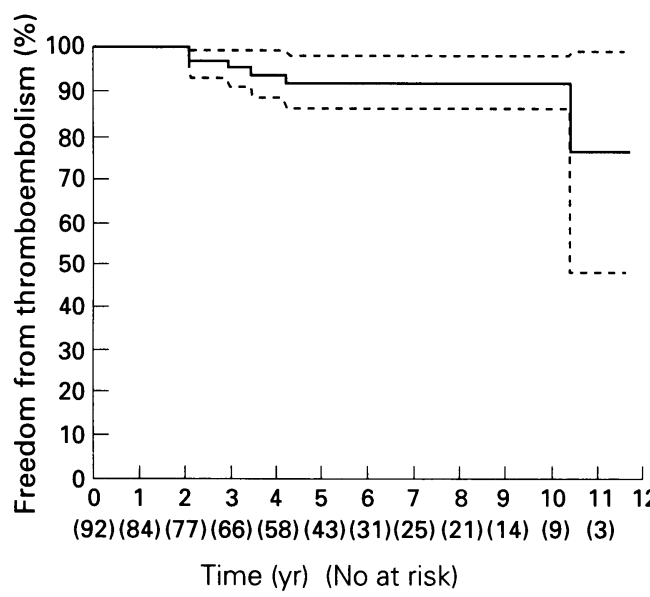

Figure 3 Actuarial freedom from thromboembolism of 92 patients who had mitral valve repair 1980-91. Broken lines are $95 \%$ CI.

\section{CLINICAL STATUS}

Of 75 survivors with completed follow up, 72 $(96 \%)$ were in NYHA functional class I or II ( $v 95 \%$ in class III or IV before operation, fig 4). An apical systolic murmur was noted in $27(37 \%)$ of 72 patients who had physical examinations at follow up. Fifty six per cent of the patients required no medications for heart failure. Sinus rhythm was present in $64 \%$ and atrial fibrillation in $36 \%$ of 59 patients who had electrocardiograms at follow up. Five patients required insertion of a permanent pacemaker because of postoperative sinus nodal dysfunction or complete atrioventricular block.

There was a significant decrease of cardiothoracic ratio after operation $(0.58(0.09) v$ $0.52(0.08), \mathrm{p}<0.01)$ in 24 patients who had a chest $x$ ray film before and after the operation.

\section{ECHOCARDIOGRAPHIC DATA}

At the end of the study, 63 (84\%) of 75 survivors had an echocardiogram at follow up. Patients who had incomplete measurements because of technical difficulties were excluded from echocardiographic analysis. Of 55 patients with a complete postoperative echocardiogram, 37 had a preoperative scan that was available for comparison. Table 8

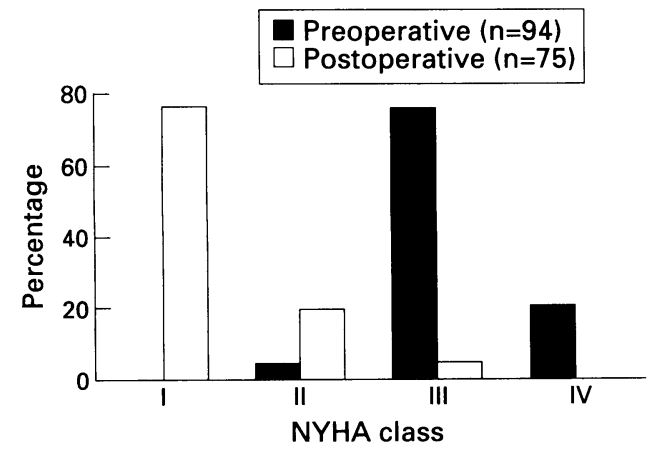

Figure 4 Distribution of NYHA functional classes before and after mitral valve repair. Distribution of postoperative and after mitral valve repair. Distribution of pos
functional classes was analysed on survivors. 
Table 8 Postoperative echocardiographic findings in 55 patients with mitral valve repair 1980-91

\begin{tabular}{lr}
\hline & No(\%) \\
\hline LVESD: & \\
Normal dimension $(\leqslant 40 \mathrm{~mm})$ & $47(85)$ \\
Increased dimension $(>40 \mathrm{~mm})$ & $8(15)$ \\
LVEDD: & $42(76)$ \\
Normal dimension $(\leqslant 56 \mathrm{~mm})$ & $13(24)$ \\
Increased dimension $(>56 \mathrm{~mm})$ & $49(89)$ \\
LVFS: & $6(11)$ \\
Normal contractility $(\geqslant 22 \%)$ & $13(24)$ \\
Reduced contractility $(<22 \%)$ & $42(76)$ \\
LAD: & $20(36)$ \\
Normal dimension $(\leqslant 40 \mathrm{~mm})$ & $23(42)$ \\
Increased dimension $(>40 \mathrm{~mm})$ & $8(15)$ \\
Mitral regurgitation: & $4(7)$ \\
No regurgitation & \\
Trivial or mild & Moderate
\end{tabular}

LVESD, left ventricular end systolic dimension; LVEDD, lef ventricular end diastolic dimension; LVFS, left ventricular fractional shortening; LAD, left atrial dimension.

shows the echocardiographic results at follow up and table 9 shows the comparison of echocardiographic measurements before and after the operation. After the operation normal left ventricular end diastolic dimension and normal left ventricular fractional shortening were shown in most of the patients. The prevalence of moderate or severe mitral regurgitation was significantly higher in patients with rheumatic disease $(50 \%$ of 16$)$ than in those with other diseases $(10 \%$ of 39 , $\mathrm{p}<0.01)$. There was a significant reduction of left ventricular end diastolic dimension after the operation.

No evidence of obstruction of the left ventricular outflow tract was detected in any of the patients. Postoperative mitral valve area was $2 \cdot 1(0.7) \mathrm{cm}^{2}$ in 48 patients who had primary valve repair. Significant mitral stenosis has developed in only one patient (mitral valve area $=1 \cdot 2 \mathrm{~cm}^{2}$ ).

PREDICTORS OF POOR OUTCOME

Stepwise logistic analysis showed that electrocardiographic evidence of previous myocardial infarction was associated with a poor outcome (odds ratio 13.7, p < 0.05). Age, NYHA functional class, aetiology of mitral valve disease, the presence of atrial fibrillation, concomitant mitral stenosis, and the performance of associated operations were not predictive of poor outcome.

\section{Discussion}

This study confirms the long-term benefit of valve repair in patients with mitral regurgitation in whom it is feasible to preserve and

Table 9 Echocardiographic measurements before and after mitral valve repair in 37 patients

\begin{tabular}{llll}
\hline & $\begin{array}{l}\text { Preoperative } \\
\text { (mean (SD)) }\end{array}$ & $\begin{array}{l}\text { Postoperative } \\
(\text { mean }(S D))\end{array}$ & $p$ Value \\
\hline LVESD (mm) & $38.0(9.3)$ & $35.7(8.4)$ & 0.12 \\
LVEDD (mm) & $61.4(7.8)$ & $52.6(8.3)$ & 0.0001 \\
LVFS (\%) & $39.5(11.4)$ & $32.4(8.3)$ & 0.0002 \\
LVEF (\%) & $68.0(12.7)$ & $61.0(11.9)$ & 0.0024 \\
LAD (mm) & $51.0(9.8)$ & $48.6(10.7)$ & 0.05 \\
\hline
\end{tabular}

LVEF, left ventricular ejection fraction; other abbreviations as for table 8 . restore the mitral apparatus. In a group of patients of varied race and including those who had additional cardiac procedures, there was satisfactory 10 year survival and similar low operative mortality to that reported in series of selected patients. ${ }^{1}$

Fewer than half of the patients required long-term anticoagulant treatment and most were free from thromboembolism at 10 years. The low incidence of bleeding complications related to anticoagulation represents some advantage over mitral valve replacement. ${ }^{36}$

The low long-term risk of infective endocarditis in patients with primary valve repair was comparable with that of other reports. ${ }^{1-3}$ The successful results are mainly attributed to the conservation of autogenous valve tissue and to the use of antibiotic prophylaxis for dental and other procedures.

Failure of mitral valve repair is generally considered at three stages. ${ }^{127}$ Immediate (operative) and early (within 2 years after surgery) valve failures usually arise because of an inadequate repair. The late recurrence of severe mitral regurgitation is more closely related to the progression of native valve disease. In this study standard techniques for intraoperative assessment of mitral valve repair were used. Visual inspection of the competence of mitral valve closure and haemodynamic assessment, particularly left atrial pressure measurement, may not exclude significant residual mitral regurgitation. Transoesophageal echocardiography, which has been shown to be more accurate in detecting mitral regurgitation after repair, ${ }^{78}$ has recently been incorporated into our intraoperative assessment. No patients in this study had immediate failure. A third of the patients who required reoperation within 2 years had moderate or severe residual mitral regurgitation after the initial repair.

The aetiology of mitral valve disease influenced the endurance of mitral valve repair. In our study patients with rheumatic disease had a higher frequency of late mitral regurgitation on Doppler analysis than those with degenerative valve disease. There was also a trend towards higher rates of reoperation in patients with rheumatic disease. Deloche reported that the rate of freedom from reoperation was significantly higher in patients with degenerative disease than those with rheumatic disease $(92 \%$ v $76 \%$ at 15 years). ${ }^{1}$ In a series of patients with myxomatous valve prolapse Cohn et al found that $85 \%$ were free from reoperation at 4 years. ${ }^{9}$

Echocardiography shows the considerable reduction in left ventricular dimensions and the normalisation of left ventricular function that occurred after mitral valve repair. It is believed that the optimal correction of valve regurgitation and the conservation of the subvalve apparatus contribute to the improvement of left ventricular function and are essential factors in the superiority of repair over replacement. ${ }^{10}$ In common with other studies we have reported that most patients have improved heart functional class and require less medication for heart failure after 
successful repair. Our study raises caution about the use of valve repair in patients with previous myocardial infarction. Previous myocardial infarction was associated with a poor outcome and a similar trend to increased operative mortality has been shown in another study. ${ }^{3}$ Mature age and NYHA functional class IV did not affect long-term prognosis.

Mitral valve repair may be feasible in over $70 \%$ of patients with mitral regurgitation. Considering the benefits afforded by intraoperative transoesophageal echocardiography, the durability of the repair and the lower rate of long-term complications, it is anticipated that mitral valves will be repaired more often in future especially in patients with degenerative disease.

We thank all the staff in the Cardiology Department, in particular Dr Peter Leslie and Dr Ron Easthope for their contribution to the follow up of patients. Dr R Luke, Dr A Mylius, and others helped review patients in other areas. Biostatistician, Dr Gordon Purdie, provided valuable advice and assistance with the statistical analysis of the data. This Wellington Area Health Board.
1 Deloche A, Jebara V, Relland J, Chauvaud S, Fabiani J, Perier P, et al. Valve repair with Carpentier techniques. P Thorac Cardiovasc Surg 1990;99:990-1002.

2 Cooper G, Wright E, Smith G. Mitral valve repair: a valuable procedure with good long term results even when performed infrequently. Br Heart f 1991;66:156-60.

3 Galloway A, Colvin S, Baumann F, Esposito R, Vohra R, Harty S. Long-term results of mitral valve reconstruction with Carpentier techniques in 148 patient with mitral insufficiency. Circulation 1988;78(suppl I):I97-105.

4 Kirklin J, Barratt-Boyes B. Cardiac Surgery. New York John Wiley, 1986:331-3

$5 \mathrm{Xu} \mathrm{M}$, McHaffie D. How is echocardiography used? An audit of 11701 studies. $N Z$ Med f 1992;105:120-2.

6 Mikaeloff $P$, Jegaden O, Ferrini M, Coll-Mazzei J, Bonnefoy J, Rumolo A. Prospective randomized study of St Jude Medical versus Bjork-Shiley or Starr-Edwards 6120 valve prostheses in the mitral position. Three hundred and fifty-seven patients operated on from 1979 to dred and fifty-seven patients operated on from 1979

7 Marwick T, Stewart W, Currie P, Cosgrove D. Mechanisms of failure of mitral valve repair: an echocardiography study. Am Heart $\mathcal{F}$ 1991;122:149-56.

8 Stewart W, Currie P, Salcedo E, Lytle B, Gill C, Schiavone W. Intraoperative Doppler color flow mapping for decisionmaking in valve repair for mitral regurgitation. Techniques and results in 100 patients Circulation 1990;81:556-66.

9 Cohn L, Disesa V, Couper G, Pamela S, Kowalker W, Collins J. Mitral valve repair for myxomatous degeneration and prolapse of the mitral valve. $f$ Thorac Cardiovasc Surg 1989;98:987-93.

10 Rahko P, Berkoff H. Echocardiographic comparison of cardiac size and function before and after surgery for isolated mitral regurgitation: superiority of mitral valye repair vs mitral valve replacement. Acta Cardiologica 1990;XLV:189-94. 